Cardiotoxicity is one of the complications following haematopoietic stem cell transplantation (HSCT), but its diagnosis may be hampered due to the presence of different post-transplant comorbidities. The aim of the study was to assess the incidence of cardiac complications and the significance of biochemical markers (NT-proBNP, ANP, ET-1, and Tnl) and ECHO systolic and diastolic parameters analysis in children treated with HSCT. Thirty consecutive children (median age 9.6 years) were included in the study. The control group consisted of 14 healthy children (median age of 10.9 years). None of the transplanted children developed clinical cardiotoxicity. Median ET-1 and NT-proBNP plasma levels were elevated when compared to controls in at least 3 out of 4 analysed time points, median ANP levels differed only in one time point, and no difference was found between median Tnl values in all analysed time points. Echocardiographic systolic parameters were within the normal range, while median E/A ratio assessed before HSCT, on day +30 , and +100 post-transplant was statistically lower in HSCT patients (respectively, 1.34, 1.37 , and 1.42 vs. 1.73). It confirms the need for careful follow up in patients who have received chemotherapy and have been treated with HSCT.

Key words: cardiotoxicity, haematopoietic stem cell transplantation, biochemical markers.

Contemp Oncol (Pozn) 2016; 20 (3): 220-224 DOI: $10.5114 /$ wo.2016.61563

\section{Can we find a good biochemical marker of early cardiotoxicity in children treated with haematopoietic stem cell transplantation?}

Agnieszka Zaucha-Prażmo ${ }^{1}$, Elżbieta Sadurska², Katarzyna Drabko', Jerzy R. Kowalczyk ${ }^{1}$

\author{
'Department of Paediatric Haematology, Oncology and Transplantology, Medical \\ University in Lublin, Lublin, Poland \\ ${ }^{2}$ Department of Paediatric Cardiology, Medical University in Lublin, Lublin, Poland
}

\section{Introduction}

Haematopoietic stem cell transplantation (HSCT) is widely used in the treatment of malignant and non-malignant disorders in paediatric patients. The procedure can be associated with severe toxicities, including cardiotoxicity [1]. Toxicities may be connected with radio- and chemotherapy used in previous treatment as well as in conditioning regimens and other potentially serious complications that may appear in the early post-transplant period. There are many different methods used to assess cardiac failure: echocardiography $(\mathrm{ECHO})$, electrocardiography, radionuclide ventriculography, or biochemical markers [2-9]. Echocardiography is one of the most commonly used non-invasive methods for imaging cardiac structure and function. Standard M-mode echocardiography allows the evaluation of motion of cardiac structures and provides accurate assessment of left ventricular systolic and diastolic parameters, which define cardiac efficiency [4]. Biochemical markers may be useful for early detection of cardiotoxicity. Atrial natriuretic peptide (ANP) is secreted by the atria in response to increased stretch as a result of an increased left atrial pressure. $\mathrm{N}$-terminal fragment of brain natriuretic peptide (NT-proBNP) is produced by ventricles in response to ventricular dilatation and increased wall stress. The levels of natriuretic peptides are inversely correlated with cardiac function [2, 7, 10-13]. Cardiac isoforms of troponin I and $\mathrm{T}(\mathrm{Tnl}, \mathrm{TnT})$ are released into the circulation rapidly after myocardial injury and may be sensitive markers of cardiac necrosis $[10,14]$. Endothelin 1 (ET-1) is a vasoconstrictor peptide synthesised in the vasculature and by the endothelial cells. Elevated plasma levels of ET-1 have been reported in association with heart failure $[11,15]$.

The aim of the study was to assess the incidence of cardiac complications and the significance of determination of NT-proBNP, ANP, ET-1, and TnI plasma levels in the early post-transplant period in children treated with HSCT.

\section{Material and methods}

Patients

A total of 30 consecutive children ( 22 boys and 8 girls) with a median age of 9.6 years (range $0.2-18$ ), in which 31 transplants were performed, were included in the study. The control group consisted of 14 healthy children with a median age of 10.9 years (range $1.6-17$ years). In the analysed group 12/31 (38.7\%) autologous (auto-HSCT), 8/31 (25.8\%) matched sibling donors (MSD), and 11/31 (35.5\%) alternative transplantations (ALT HSCT: 9 matched unrelat- 
ed donor - MUD and 2 mismatched family donor - MMFD) were performed. 23/30 (76.7\%) patients were transplanted because of malignant diseases: 11 had acute leukaemias (ALL-4, AML-7); 9 - solid tumours (neuroblastoma - 2, Ewing sarcoma -4 , rhabdomyosarcoma - 2, germ cell tumour - 1) and 3 children were treated for lymphomas. Seven transplantations (23.3\%) were performed in children with non-malignant disorders (severe aplastic anaemia - 4, myelodysplastic syndrome - 1, Wiscott-Aldrich Syndrome - 1 , Ommen syndrome -1 , adrenoleukodystrophy -1 ). Conditioning regimens differed according to the diagnosis and are presented in Table 1.

Patients transplanted for malignant diseases were previously treated with anthracyclines, according to standard chemotherapy protocols, in a median dose of $240 \mathrm{mg} / \mathrm{m}^{2}$ $\left(180-360 \mathrm{mg} / \mathrm{m}^{2}\right)$. All patients were managed according to the institutional protocol for HSCT. Fluid balance was carefully monitored and compensated with fluids or diuretics as clinically indicated. Patients undergoing allogeneic HSCT received graft versus host disease (GVHD) prophylaxis with cyclosporine +/- short methotrexate. Treatment of aGvHD followed EBMT guidelines [16].

\section{Methods}

The plasma levels of biochemical markers: NT-proBNP, ANP, Tnl, and ET-1, were measured before HSCT and every week for three weeks post-transplant (on days $-7,+7,+14$, and +21 ). The values of serum cardiac troponin I were assessed immediately in the Central Lab by Immunoassay (Abbot). Blood samples for assessing NT-proBNP, ANP, and ET-1 were collected from the patients on the analysed days then immediately centrifuged and separated and the plasma was stored at $-80^{\circ} \mathrm{C}$ until the analysis was performed. The samples were thawed and analyzed at the same time. The values of NT-proBNP, ANP, and ET-1 were estimated by Electrochemiluminescent Immunoassay (Biomedica). Left ventricular systolic parameters [fractional shortening (FS) and ejection fraction (EF)] and diastolic parameters [early peak flow velocity/atrial velocity (E/A ratio) and isovolumic relaxation time (IVRT)] were measured using M-mode standard echocardiography prior to HSCT and approximately day +30 and +100 after transplantation. The same biochemical markers, as well as echocardiographic parameters, were assessed once in the control group.

Statistical analysis was performed using computer software Statistica 10.0 Stat Soft Inc. (USA). The statistical difference between biochemical marker values in patients and controls was compared using non-parametric Mann-Whitney $U$ test. For the comparison of the values of chosen parameters in more than two groups the Kruskal-Wallis test was used. The correlation between assessed parameters was estimated using Pearson's test. The statistical significance level of $p<0.05$ was assumed.

The study was approved by the Ethical Committee of the Medical University of Lublin, Poland.

\section{Results}

None of the children from the analysed group developed clinical cardiotoxicity in the post-transplant period.
Baseline echocardiographic systolic parameters were within the normal range in all patients included in the study (median FS - 40.2\%, median EF - 73.3\%). Concerning diastolic parameters, the median E/A ratio assessed before HSCT was statistically lower in the transplanted patients (1.34 vs. 1.73, $p<0.05$ ) as well as median E/A on day +30 (1.37 vs. $1.73, p<0.05)$ and on day +100 (1.42 vs. 1.73, $p<0.05)$ post-transplant. Median SF and EF values on day +30 in transplanted children and in the control group did not differ significantly. The results are presented in Table 2 .

Median ET-1 values in the transplanted children were significantly higher than estimated in controls in all analysed time points, and median NT-proBNP values differed significantly at three analysed time points: before HSCT (on day -7$)$ and on days +7 and +14 post-transplant. Comparing median ANP values in transplanted patients and controls, a statistically significant difference was found only on day +14 post-transplant. No difference was found between the median Tnl values in all analysed time points. Median values of all analysed biochemical parameters (ET-1, ANP, NT-proBNP, TNI) are presented in Table 3.

Table 1. Conditioning regimens in transplanted patients according to the type of transplant and diagnosis

\begin{tabular}{|c|c|c|}
\hline $\begin{array}{l}\text { Type of } \\
\text { transplant } \\
n(\%)-\text { all } \\
\text { transplants } \\
n=31(100 \%)\end{array}$ & Diagnosis $(n)$ & $\begin{array}{l}\text { Conditioning } \\
\text { regimens }(n)\end{array}$ \\
\hline \multirow{4}{*}{$\begin{array}{l}\text { Auto } \\
n=12(38.7 \%)\end{array}$} & RMS (2), GCT (1) & Mel, Eto, Carbo (3) \\
\hline & $\begin{array}{c}\text { Sa Ewing (4), AML (1), } \\
\text { NBL (2) }\end{array}$ & Bu, Mel (7) \\
\hline & ALL & TBI, Eto (1) \\
\hline & AML & Treo, Mel (1) \\
\hline \multirow{6}{*}{$\begin{array}{l}\text { Allo MSD } \\
n=8(25.8 \%)\end{array}$} & lymphoma & TBI, Thiot, Eto (2) \\
\hline & ALL & TBI, Eto (2) \\
\hline & SAA & Cy (1) \\
\hline & WAS & Flu, Mel (1) \\
\hline & $A D L X$ & Flu, Mel, Thiot (1) \\
\hline & AML & $\mathrm{Bu}, \mathrm{Cy}(1)$ \\
\hline \multirow{7}{*}{$\begin{array}{l}\text { Allo alternative } \\
\text { (MUD, MMFD) } \\
n=11(35.5 \%)\end{array}$} & ALL (1), lymphoma (1) & TBI, Eto (2) \\
\hline & $\mathrm{AML}$ & TBI, Flu (1) \\
\hline & AML & Treo, Cy, Mel (3) \\
\hline & SAA & Flu, Cy (2) \\
\hline & SAA II & TBI (1) \\
\hline & MDS & Bu, Cy, Mel (1) \\
\hline & Omenn syndrome & Flu, Mel (1) \\
\hline
\end{tabular}

Auto - autologous transplantation; Allo alternative - transplantation from alternative donors; MSD - matched sibling donor; MUD - matched unrelated donor; MMFD - mismatched family donor; TBI - total body irradiation; Thiot - thiotepa; Eto - etoposide; Flu-fludarabine; Bu-busulfan; Mel-melfalane; Treo - treosulfan; Cy - cyclophosphamide; Carbo - carboplatinum; AML acute myeloblastic leukaemia; ALL - acute lymphoblastic leukaemia; SAA - severe aplastic anaemia; Sa Ewing - Ewing sarcoma; NBL - neuroblastoma; WAS - Wiscott-Aldrich syndrome; ALD-X-adrenoleukodystrophy; RMS rhabdomyosarcoma; GCT - germ cell tumour 
Table 2. Echocardiographic systolic and diastolic parameters at analysed time points in patients and controls

\begin{tabular}{|c|c|c|c|c|}
\hline \multirow[t]{2}{*}{ ECHO parameter } & \multirow[t]{2}{*}{ Time point } & $\begin{array}{c}\text { Analysed patients } \\
\qquad n=31\end{array}$ & $\begin{array}{c}\text { Controls } \\
n=14\end{array}$ & $p$-value \\
\hline & & Median (range) & Median (range) & \\
\hline LVSF (\%) & Before HSCT & $40.2(28.6-50.5)$ & $40.85(36.0-57.2)$ & NS \\
\hline LVEF (\%) & Before HSCT & $73.3(55.5-82.0)$ & $71.0(67.1-83.7)$ & NS \\
\hline LVSF (\%) & Day +30 & $37.0(27.9-49.5)$ & $40.85(36.0-57.2)$ & NS \\
\hline LVEF (\%) & Day +30 & $69.2(55.2-82.0)$ & $71.0(67.1-83.7)$ & NS \\
\hline LVSF (\%) & Day +100 & $42.1(30.3-51.2)$ & $40.85(36.0-57.2)$ & NS \\
\hline LVEF (\%) & Day +100 & $74.1(60.0-83.7)$ & $71.0(67.1-83.7)$ & NS \\
\hline$E / A$ & Before HSCT & $1.34(0.76-2.62)$ & $1.73(1.47-2.57)$ & 0.00015 \\
\hline IVRT (ms) & Before HSCT & $63(49-83)$ & $65(50-77)$ & NS \\
\hline$E / A$ & Day +30 & $1.37(0.76-2.62)$ & $1.73(1.47-2.57)$ & 0.0005 \\
\hline IVRT (ms) & Day +30 & $63(48-83)$ & $65(50-77)$ & NS \\
\hline$E / A$ & Day +100 & $1.42(1.26-1.72)$ & 1.73 (1.47-2.57) & 0.0002 \\
\hline IVRT (ms) & Day +100 & $62(47-82)$ & $65(50-77)$ & NS \\
\hline
\end{tabular}

Significant $p$ value $<0.05$

HSCT - haematopoietic stem cell transplantation; LVEF - left ventricular ejection fraction; LVFS - left ventricular shortening fraction; E/A ratio - early peak flow velocity/atrial velocity; IVRT - isovolumic relaxation time

Table 3. Biochemical parameters at analysed time points in patients and controls

\begin{tabular}{|c|c|c|c|c|}
\hline \multirow[t]{2}{*}{$\begin{array}{l}\text { Biochemical } \\
\text { parameter }\end{array}$} & \multirow[t]{2}{*}{ Time point } & $\begin{array}{l}\text { Analysed patients } \\
\qquad n=31\end{array}$ & $\begin{array}{c}\text { Controls } \\
n=14\end{array}$ & \multirow[t]{2}{*}{$p$-value } \\
\hline & & Median (range) & Median (range) & \\
\hline \multirow[t]{4}{*}{$\mathrm{ET}-1[\mathrm{fmol} / \mathrm{ml}]$} & -7 & $0.43(0.07-2817)$ & $0.22(0.09-0.63)$ & 0.002 \\
\hline & +7 & $0.52(0.15-424)$ & & $<0.001$ \\
\hline & +14 & $0.40(0.07-1481)$ & & 0.001 \\
\hline & +21 & $0.48(0.49-1635)$ & & 0.005 \\
\hline \multirow{4}{*}{$\begin{array}{l}\text { NT-proBNP } \\
{[\mathrm{fmol} / \mathrm{ml}]}\end{array}$} & -7 & $13.27(0.55-128.8)$ & $3.31(0.74-19.06)$ & 0.016 \\
\hline & +7 & $10.3(0.78-107.4)$ & & 0.015 \\
\hline & +14 & $12.39(0.63-564.2)$ & & 0.006 \\
\hline & +21 & $7.78(0.15-85.9)$ & & NS \\
\hline \multirow[t]{4}{*}{ ANP [fmol/l] } & -7 & $1110(280-5001)$ & 1064 (298-2705) & NS \\
\hline & +7 & $1287(307-6589)$ & & NS \\
\hline & +14 & $1700(101-7325)$ & & 0.022 \\
\hline & +21 & 1203 (266-7310) & & NS \\
\hline \multirow[t]{4}{*}{ Tnl [ng/ml] } & -7 & $<0.01(0.0-2.14)$ & $0.01(0.0001-0.02)$ & NS \\
\hline & +7 & $<0.01(0.0-1.64)$ & & NS \\
\hline & +14 & $<0.01(0.0-1.11)$ & & NS \\
\hline & +21 & $<0.01(0.0-0.07)$ & & NS \\
\hline
\end{tabular}

Significant $p$-value $<0.05$

ET-1 - endothelin 1; NT- proBNP - brain natriuretic peptide; ANP - atrial natriuretic peptide; $T n 1$ - troponin I

Since median ET-1 and NT-proBNP plasma levels were elevated in at least 3 out of 4 analysed time points in the transplanted patients when compared to controls, the analysis was extended according to the type of transplant. No difference was found in median ET-1 and NT-proBNP values depending on the particular type of transplant when compared with each other, while median EF-1 and NT-proBNP values in auto, MSD, and especially in alternative transplant recipients differed signifi- cantly in most analysed time points when compared to controls. Analyses and values are presented in Table 4.

Dependences between median biochemical marker values and echocardiographic systolic and diastolic parameters were analysed. No correlation was found, except for median ET-1 level assessed on day +14 and SF and EF assessed on day $+30(r=-0.374$ and -0.446 , respectively) and on day $+100(r=-0.420$ and -0.477 , respectively). 
Table 4. The median values of endothelin-1 and NT-pro BNP at all analysed time points according to the type of transplant

\begin{tabular}{|c|c|c|c|c|c|c|}
\hline \multirow[t]{2}{*}{ Parameter } & \multirow[t]{2}{*}{ Time point } & \multicolumn{3}{|c|}{ Type of transplant } & \multirow[t]{2}{*}{ Controls } & \multirow[t]{2}{*}{$p$-value } \\
\hline & & Auto & Allo MUD, MMFD & Allo MSD & & \\
\hline \multirow{4}{*}{$\begin{array}{l}\text { Endothelin -1 } \\
{[\mathrm{fmol} / \mathrm{ml}]} \\
\text { median, (range) }\end{array}$} & -7 & $0.54(0.07-1.82)$ & $0.31(0.15-2815)$ & $0.43(0.21-1.12)$ & \multirow{4}{*}{$\begin{array}{c}0.22 \\
(0.09-0.63)\end{array}$} & 0.03 \\
\hline & +7 & $0.48(0.22-1.15)$ & $0.52(0.15-424)$ & $0.49(0.25-11.12)$ & & 0.004 \\
\hline & +14 & $0.49(0.26-2.5)$ & $0.40(0.07-1481)$ & $0.39(0.15-10.39$ & & 0.007 \\
\hline & +21 & $0.49(0.199-1.71)$ & $0.58(0.05-1635)$ & $0.399(0.21-1.12)$ & & NS \\
\hline \multirow{4}{*}{$\begin{array}{l}\text { NT-proBNP } \\
{[\mathrm{fmol} / \mathrm{ml}]} \\
\text { median (range) }\end{array}$} & -7 & $6.49(0.65-63.69)$ & $16.06(0.55-128.8)$ & $12.78(3.17-59.77)$ & \multirow{4}{*}{$\begin{array}{c}3.31 \\
(0.74-19.06)\end{array}$} & NS \\
\hline & +7 & $4.13(0.78-67.22)$ & $59.35(6.35-107.4)$ & $16.86(0.99-64.12)$ & & 0.0007 \\
\hline & +14 & $7.93(0.64-32.97)$ & $24.26(7.38-564.2)$ & $14.45(4.26-46.65)$ & & 0.008 \\
\hline & +21 & $7.57(0.15-17.08)$ & $10.58(2.32-85.9)$ & $6.37(2.13-19.6)$ & & 0.26 \\
\hline
\end{tabular}

Auto - autologous transplantation; MUD - matched unrelated donor; MMFD - mismatched family donor; MSD - matched sibling donor

In 9 out of 19 allogeneic transplant recipients (47.4\%) aGvHD was diagnosed ( $1^{\text {st }}$ grade in two patients, $2^{\text {nd }}$ grade in six patients, and $3^{\text {rd }}$ grade in one patient). No difference in echocardiographic parameters and in biochemical marker levels was found between patients with and without aGVHD. None of the patients developed acute kidney injury. Of all the analysed group, 10 patients died (33.3\%): seven because of disease relapse/progression, one patient developed multiorgan failure, one because of graft rejection, and one because of infectious complications.

\section{Discussion}

Clinically significant cardiotoxicity after HSCT has been estimated in about $5 \%$ of adult patients [7]. In patients following HSCT, myocardial function can be impaired by cardiotoxic drugs used in conditioning regimens, which can cause toxic endothelial damage [3, 5]. Early diagnosis of cardiac failure in patients following HSCT may be difficult because they may have multiple medical problems: veno-occlusive disease (VOD), acute renal failure, GvHD, infections [17]. Heart failure may also be due to the use of hyperhydration regimens, transfused blood components, impaired renal function, sepsis, or electrolyte abnormalities [18]. Previous treatment of malignancy with chemotherapeutic agents, including anthracyclines, may explain our results, in which the median E/A ratio was lower and median ET-1 and NT-pro BNP values were elevated before HSCT in the analysed group. NT-proBNP elevations may reflect chemotherapy-associated diastolic abnormalities, and the level may be increased by myocardial stress without necrosis. ET-1 is a biomarker of vascular injury and is increased in many conditions in which vascular endothelium is vulnerable. Endothelial cells upregulate ET-1 secretion in response to hypoxia, oxidized LDL, pro-inflammatory cytokines, and bacterial toxins [9, 12] Several studies confirm the usefulness of ET-1 and NTproBNP as predictors of chemotherapy related cardiotoxicity [14, 19-24]. The study by Hakayawa, performed on a group of 34 children receiving doxorubicin-containing chemotherapy, showed natriuretic peptides to be useful markers of cardiotoxicity in children [19]. In studies by Nouisiainen, conducted in adult patients with Hodgkin's lymphoma receiving high doses of doxorubicin (400-500 $\mathrm{mg} / \mathrm{m}^{2}$ ), increased concentrations of plasma natriuretic peptides were found $[23,24]$. ET-1 may also contribute to the prediction of doxorubicin-induced cardiotoxicity, which was shown in Yamashita's study, performed on patients with breast and lung cancer, in which ET-1 plasma concentrations rose progressively during doxorubicin treatment [25]. Measurement of natriuretic peptides plasma levels has already been used to monitor cardiotoxicity following HSCT. Snowden confirmed the utility of plasma BNP assessment as a ventricular dysfunction indicator in adult patients [2]. Kuittinen studied patients with non-Hodgkin lymphoma and observed symptoms of cardiotoxicity diagnosed after conditioning treatment, and a correlation was found between the changes in NTpro BNP levels and LVEF, and more strongly with diastolic function parameters [18]. Morandi et al., in their study, concluded that use of high-dose cyclophosphamide, but also cytarabine, mitoxantrone, melphalan, and fludarabine may induce cardiac injury [7]. In our analysis median NT-proBNP levels were statistically higher on days +7 and +14 post-transplant, as well as when assessed before HSCT. According to our study, only plasma levels of ET-1 assessed in the early post-transplant period inversely correlated with systolic echocardiographic parameters.

Our results concerning the diagnostic values of ANP and $\mathrm{Tnl}$ as predictors of cardiotoxicity do not confirm their usefulness. In previous studies, e.g. in the Herman et al. study on rats, in which doxorubicin was given, troponin was proven to be useful in early detection of cardiotoxicity [14]. In our study no difference was found between the median Tnl values at all analysed time points in transplanted patients compared to controls. The results are similar to those obtained by Mavinkurve-Grothuis et al. in their analysis conducted on childhood cancer survivors, in which abnormal troponin levels were not found in any of the patients [21]. Also in the study by Romano et al., performed on 34 breast cancer patients, Tnl was abnormal only occasionally in four of them, and in the retrospective Sherief et al. study none of the analysed survivors of acute leukaemia had abnormal troponin levels [20, 22].

In our cohort of patients, clinical symptoms of cardiac dysfunction were not observed, but abnormalities in biochemical marker levels, especially ET-1 and NTproBNP, as well as in diastolic echocardiographic parameters, were found. This confirms the need for careful follow-up in pa- 
tients who have received chemotherapy and have been treated with haematopoietic stem cell transplantation.

The authors declare no conflict of interest.

\section{References}

1. Eames GM, Crosson J, Steinberger J, et al. Cardiovascular function in children following bone marrow transplant: a cross-sectional study. Bone Marrow Transplant 1997; 19: 61-6.

2. Snowden JA, Hill G, Hunt P, et al. Assessment of cardiotoxicity during haematopoietic stem cell transplantation with plasma brain natriuretic peptide. Bone Marrow Transplant 2000; 26: 309 13.

3. Murdych T, Weisdorf DJ. Serious cardiac complications during bone marrow transplantation at the University of Minnesota, 1977-1997. Bone Marrow Transplant 2001; 28: 283-7.

4. Jiji RS, Kramer CM, Salermo M. Non-invasive imaging and monitoring cardiotoxicity of cancer therapeutic drugs. J Nucl Cardio 2012; 19: 377-88.

5. Tichelli A, Bhatia S, Socie G. Cardiac and cardiovascular cosequences after haematopoietic stem cel transplantation. $\mathrm{Br} J \mathrm{He}$ aematol 2008; 142: 11-26.

6. Horacek JM, Pudil R, Tichy M, et al. The use of biochemical markers in cardiotoxicity monitoring in patients treated for leukemia. Neoplasma 2005; 52: 430-4.

7. Morandi P, Ruffini PA, Benvenuto GM, et al. Cardiac toxicity of highdose chemotherapy. Bone Marrow Transplant 2005; 35: 323-8.

8. Brockstein BE, Smiley C, Al-Sadir J. Cardiac and pulmonary toxicity in patients undergoing high-dose chemotherapy for lymphoma and breast cancer: prognostic factors. Bone Marrow Transplant 2000; 25: 885-91.

9. Dudka J, Burdan F, Korga A, et al. The diagnosis of anthracycline-induced cardiac damage and heart failure. Postępy Hig Med Dosw 2009; 63: 225-33.

10. Lipshultz SE, Rifai N, Sallan SE, et al. Predictive value of cardiac troponin $\mathrm{T}$ in pediatric patients at risk for myocardial injury. Circulation 1997; 96: 2641-8.

11. Wei CM, Lerman A, Rodeheffer RJ, et al. Endothelin in human congestive heart failure. Circulation 1994; 89: 1580-6.

12. Tikanoja T, Riikonen P, Perkkio M, et al. Serum N-termina atrial natriuretic peptide (NT-ANP) In the cardiac follow-up in children with cancer. Med Pediatr Oncol 1998; 31: 73-8.

13. Suzuki T, Hayashi D, Yamazaki T, et al. Elevated B-type natriuretic peptide levels after anthracycline administration. Am Heart 1998; 136: 362-3.

14. Herman EH, Zhang J, Lipshultz SE, et al. Correlation between serum levels of cardiac troponin $T$ and the severity of the chronic cardiomyopathy induced by doxorubicin. J Clin Oncol 1999; 17: 2237-43.

15. Gillespie HS, McGann CJ, Wilson BD. Noninvasive diagnosis of chemotherapy related cardiotoxicity. Current Cardiol Rev 2011; 7: 234-44.

16. Apperly J, Masszi T. Graft-versus-host disease. In: Apperly J, Carreras E, eds. Haematopoietic Stem Cell Transplantation. Revised edition 2012; 217-233.

17. Carreras E. Early complications after HSCT. In: Apperly J, Carreras E (eds.). Haematopoietic Stem Cell Transplantation. Revised edition 2012; 177-195.

18. Kuittinen T, Husso-Saastamoinen M, Sipola P, et al. Very acute cardiac toxicity during BEAC chemotherapy in non-Hodgkin's lymphoma patients undergoing autologous stem cell transplantation. Bone Marrow Transplant 2005; 36: 1077-82.

19. Hayakawa H, Komada Y, Hirayama M, et al. Plasma levels of natriuretic peptides in relation to doxorubicin-induced cardiotoxicity and cardiac function in children with cancer. Med Ped Oncol 2001; 37: 4-9.
20. Romano S, Fratini S, Ricevuto E, et al. Serial measurements of NT-proBNP are predictive of not-high dose anthracycline cardiotoxicity in brest cancer patients. Br J Cancer 2011; 22: 1663-8.

21. Mavinkurve-Grothius AM, Groot-Loonen J, Bellersen L, et al. Abnormal NT-pro-BNP levels in asymptomatic long-term survivors of childhood cancer treated with anthracyclines. Ped Blood Cancer 2009; 52: 631-6.

22. Sherief LM, Kamal AG, Khalek EA, et al. Biomarkers and early detection of late onset anthracycline-induced cardiotoxicity in children. Hematology 2012; 17: 151-6.

23. Nouisiainen T, Jantunen E, Remes J, et al. Natriuretic peptides as markers of cardiotoxicity during doxorubicin treatment for non-Hodgkin's lymphoma. Eur J Haematol 1999; 62: 135-41.

24. Nousiainen T, Vanninen E, Jantunen E, et al. Natriuretic peptides during the development of doxorubicine-induced left ventricular diastolid dysfunction. J Intern Med 2002; 251: 228-34.

25. Yamashita J, Ogawa M, Shirakusa T. Plasma endothelin-1 as a mark er for doxorubicin cardiotoxicity. Int J Cancer 1995; 62: 542-7.

\section{Address for correspondence}

\section{Agnieszka Zaucha-Prażmo PhD}

Department of Paediatric Haematology, Oncology

and Transplantology

Medical University in Lublin,

Gębali 6

20-093 Lublin, Poland

e-mail: a.prazmo@umlub.pl

Submitted: 3.02 .2015

Accepted: $\quad 15.10 .2015$ 